\title{
Improving norms in research culture to incentivize transparency and rigor
}

\author{
David Mellor \\ Director of Policy, Center for Open Science \\ https://orcid.org/0000-0002-3125-5888
}

This paper was accepted for publication in Educational Psychologist volume 56, issue 2; scheduled for April 2021, as a part of a special issue titled, "Educational psychology in the open science era."

This preprint has been peer reviewed, but not copy edited by the journal and may differ from the final published version.

\begin{abstract}
Improving research culture to value transparency and rigor is necessary to engage in a productive "Credibility Revolution." The field of educational psychology is well positioned to act toward this goal. It will take specific actions by both grassroots groups plus leadership to set standards that will ensure that getting published, funded, or hired is determined by universally supported ideals. These improved standards must ensure that transparency, rigor, and credibility are valued above novelty, impact, and incredibility. Grassroots groups advocate for change and share experience so that the next generation of researchers have the experience needed to sustain these early moves. Each community can take inspiration from others that have made shifts toward better practices. These instances provide opportunities for emulating trail-blazers, training for new practices such as preregistration, and constructively evaluating or criticizing practice in ways that advances the reputation of all involved.
\end{abstract}

\section{Introduction}

If you ask ten scientists for a professional opinion, you will receive ten different responses (see Silberzahn et.al, 2018, below for a demonstration of this truism). There are, however, a few areas of near consensus, one of them being that science should proceed with collective possession of scientific goods or knowledge. Without such possession of these goods by the scientific community, progress occurs in secrecy, without sharing and without the benefits that 
accrue from such cooperation, such as verification of results and building upon recent advances. Anderson and her colleagues found that over $95 \%$ of researchers surveyed agreed with this sentiment (2007), that science should proceed according to this and other "Mertonian Norms:" universalism, disinterestedness, and organized skepticism (Merton, 1973). A slightly weaker consensus was shown when she asked researchers to report how they themselves act in accordance with, or opposition of, these norms. Notably, however, when asked about how others act, there was a widespread sense that they do not act in accordance with these norms, but rather with counternorms of secrecy, dogmatism, and of valuing quantity over quality. This tension between the ideal ways in which one desires to act and the perceived ways in which other scholars are actually acting sets up a powerful collective action problem. Acting with more transparency than is expected by norms, required policies, or anticipated by peers sets up a challenging tension between expected community and individual interest. It creates a conflict in which one's career prospects are perceived to be at stake, and a collective action problem wherein each stakeholder waits for steps to be taken by others. It is in this challenging environment that we seek to foster change and improve the culture of scientific practice.

One might wonder why such change would even be desirable for educational psychologists. Put simply, there is an urgent need to improve the trust and rigor of research. Data underlying reported findings is rarely available for verification or reuse (Gabelica et al., 2019; Stodden, et al. 2018). Anonymous surveys across multiple disciplines report alarmingly high rates of "questionable research practices" (QRPs) that invalidate many results (Agnoli et al., 2017; Fraser et al., 2018; John et al., 2012; Rabelo et al., 2019). Some of these QRPs, on their own, appear to be relatively innocuous. For example, only reporting the results of tests that "worked" (i.e. resulted in statistically significant findings) or continuing to sample a population until a significant result occurs. However, these and similar practices undermine the credibility and findings, increase the chance of reporting false positives that could have long lasting costs in terms of policies or education practices enacted, and lead to results that cannot be reproduced. Consider, as an example, the results of the "red card study" by Silberzahn and colleagues (2018). In this work, 29 teams of data analysts were asked to answer the research question: "Whether soccer referees are more likely to give red cards to dark-skin-toned players than to light-skin-toned player." They were then given the same dataset to answer that question. With the same research question and the same data, one might expect that there would be one answer, perhaps with a reasonable band of uncertainty. There were, however, 29 different 
answers. Answers ranged from showing no difference to nearly three times more likely ${ }^{1}$, and the statistical significance and measure of uncertainty varied widely between data analysts. See also Simmons, et. al. (2011) and loannidis (2005) for a description of how practices such as those admitted to by many researchers lead to an increase in the number of false positives reported in the scientific literature.

The fact that such flexibility in data analysis exists is not problematic, or at least not the source of problems. This flexibility is inevitable in a complicated world, particularly in field situations such as classrooms. Rather, problems arise when there is a preference for one type of result, the ability to find that type of result no matter the data, and the self-admitted prevalence of such behaviors. And that preference for such results does indeed exist in the form of publication bias, which has been well documented for decades (Atkinson, Furlong, \& Wampold, 1982; Franco, Malhotra, \& Simonovits, 2014; Mahoney,1977; Sterling, 1959). See Gehlbach \& Robinson (2021/this issue) for an overview of how these practices lead to irreproducible findings.

Traditionally we have relied on the peer review process to catch and address these issues. However, I would argue that it is not sufficient for addressing the problems. The process of peer review is not set up to verify reported findings. Indeed, it is too much to expect volunteer reviewers to verify the reported statistical analyses on their own, or to re-analyze the data collected by the authors. It is simply not designed to "check under the hood" to see how work was actually conducted. And even if there is a will to do this, it is only possible to do so on the materials that are presented. In a culture where transparency is not the norm, peer review is limited to the claims that are reported in the manuscript, not on the years of work that occurred before a single word was written. Peer review should not be abandoned, but we have to find a way to make verification possible, desirable, and straightforward. The final possible safeguard in the existing ecosystem is replicating empirical claims. And as is discussed in Plucker and Makel, 2021/this issue, the barriers to such work are high, leading to few such direct replications (Makel et al., 2012).

The cumulative effect of lack of replication, bias in reporting, preference for particular outcomes, and the ability to preferentially obtain these outcomes is a body of empirical research findings that largely cannot be reproduced (verified through conducting the original analysis with the original data; Chang et al., 2015, Hardwicke et al., 2018; loannidis et al., 2009) or replicated (verified through conducting the original analysis with newly collected data; C. F. Camerer et al.,

\footnotetext{
${ }^{1}$ For a fantastic summary of this work and similar issues, see Christie Aschwanden's reporting in 538, published in 2015 https://fivethirtyeight.com/features/science-isnt-broken.
} 
2016; Colin F. Camerer et al., 2018; Cova et al., 2018; OSC, 2015). Even bodies of research that have been supported through existing standards of evidence, broad use of meta-analyses, and many conceptual replications can sometimes not be replicated (e.g Hagger et al., 2016).

The above points, and those mentioned in the accompanying articles of this issue, establish means, motive, and opportunity for questionable research practices. These practices can lead to schools enacting initiatives and policies that rely on evidence that is, at best, weak and at worst, illusory. We cannot alter the means (we will always have complex questions and data, indeed that is an important factor to strive for). However, we can and should reduce the motives and opportunity for such behaviors, and that is where open science practices become especially important to the educational psychologist.

Improving research culture to normalize maximum transparency is challenging. However, there is a roadmap for the adoption of new, better practices. In Diffusion of Innovations, Rogers (1963) described how, time and again, people adopt new technologies according to a normal distribution of personality traits. Innovators make up a small portion of society and are the first to take up new ideas. Early adopters and the early majority make up increasingly large portions of society and account for a majority of individuals. Late adopters finally pick up the new ideas once they are common. Finally, there are a small number of laggards, who will hold on to older techniques until they are no longer feasible. Each group responds to different opportunities or incentives when presented with the new practices, which can be implemented by different stakeholders.

Creating a strategy for changing a culture requires providing the right opportunity for the right person. Innovators only need a new practice to be possible in order to pick it up and see for themselves whether it is an improvement on the status quo (see Figure 1). Early adopters will find these new practices, but they must be designed to be easy in order to encourage uptake. The early majority can respond best when a community of practice develops around them so that they can see and learn from their colleagues adopting these new norms. A late majority can be characterized by those who need specific rewards for such actions, and laggards will come on board only when required to do so (Nosek, 2019).

[Figure 1] 
Making ideal scientific practices possible, easy, normative, rewarding, and required is feasible through concerted efforts by individuals and institutions. What follows is a specific set of examples and recommendations for each of the stages of this roadmap for culture change. These recommendations are by no means exhaustive, but they have been gaining traction across a number of disciplines in part due to support from organizations like the Center for Open Science. The Center provides free information and resources that support scholars engaging in open science practices.

\section{Make it Possible}

The foundation of this strategy requires that a particular action is possible. Making it possible is sufficient for those innovators who are early adopters and highly motivated to take a step, no matter the barriers.

For many open science practices, "Making it possible" is achieved through a digital repository of research materials. These repositories are typically designed to archive items that are not commonly preserved or rarely published, such as protocols, stimuli, questionnaires, raw data, analytical code, and early versions or preliminary findings. Better still, this can occur through version-controlled documentation of methods on electronic notebooks, specification of study ideas before, during, and after data collection, and preservation of study items such as data and analytical code through use of a persistent, unique identifier (PUID). These PUIDs make later identification of the precise dataset or research instrument easier for the reader, which in many cases may be a member of the original team working to expand their previous work. The Registry of Research Data Repositories (https://www.re3data.org/) catalogues over 2,000 repositories into which data and digital research materials can be posted. Each of these can serve the research by providing an end-of-life preservation of research materials.

In education research, such quick and easy data sharing can be particularly challenging (see Fleming et al., 2021/this issue for a more comprehensive discussion on challenges and opportunities in education research on this topic). In a recent unconference on promoting open practices in education research (https://osf.io/da3nf/), hundreds of participants provided opportunities and challenges for such transparency (https://osf.io/425gn/). These frequently note that complete transparency is not ethical or legally feasible. Fortunately, there are workflows that help overcome some of these barriers. For example, there are over 1,100 repositories listed at $\mathrm{Re} 3$ data that provide some form of restricted access for sensitive data sets that cannot be 
made publicly available but that can still be preserved for other research uses with some form of ethical check prior to access (readers can use the "restricted access" data filter in the database). There are databases that even make ethical sharing of qualitative data possible, such as the Qualitative Data Repository at Syracuse University (http://qdr.syr.edu/).

Even if sharing data in such a protected access repository, it is important and possible to take steps to do so mindfully. Meyer (2018) provides practical steps to consider when that occurs, such as how to plan for this step, making sure informed consent is truly informed, and steps to minimize risk. And when data sharing is simply not possible, the code, instruments, surveys, and analytical code are all still valuable items that can and should be shared with few, if any, ethical considerations so that later reproducibility becomes more possible. Likewise, taking guidance from recommended journal policies in the TOP Guidelines (cos.io/top) provides instructions for what to do when such data transparency is simply impossible. These include:

A. Explain the restrictions on the dataset or materials and how they preclude public access.

B. Provide a public description of the steps others should follow to request access to the data or materials.

C. Provide software and other documentation that will precisely reproduce all published results.

D. Provide access to all data and materials for which the constraints do not apply.

Taking such steps confer many of the overall benefits of transparency: allowing researchers to better document and reuse their own working materials and increasing trust and credibility in the presented findings.

\section{Make it Easy}

An end-of-life repository is useful for preservation. However, a better system would include a workspace for conducting research that is then directly connected to places where open science practices can be preserved: a registry, data repository, and preprint server to connect and preserve the items that are normally lost after publication of final results. Such a system can serve the short-term interests of the research team (i.e. helping to manage ongoing work in research labs), while satisfying the long term preservation needs of the community. For educational researchers, this approach should be a better strategy for improving documentation and preservation-requiring little additional curation or documentation to occur from the end of a project to submission for publication. The Open Science Framework (OSF, https://osf.io), 
provides an example of this type of open source tool. Focused around project pages designed to organize ongoing projects in research labs, the OSF can provide persistent, citable links to project materials timestamped to show when they were developed and submitted during the project. Through this tool, authors no longer need to reorganize materials at the end of a project, when many academics are in a rush to publish and already thinking about their next study.

Deciding to take an action is followed by the inevitable barrier of knowing how best to achieve that action. As mentioned below, seeing examples of shared datasets, preregistrations, or other open practices completed by peers is one way to learn the basic steps. Another is to seek out training materials, primers, or guidelines for the action. One growing resource that can be helpful to the educational psychology community is the Open Scholarship Knowledgebase (OSKB), which has a growing, curated list of such materials (https://www.oercommons.org/hubs/OSKB). The OSKB is organized in multiple ways so as to maximize discoverability of resources. First by resource type (such as data, educational materials, items related to reproducibility) and then by user need (such as researcher, funder, or editor), the OSKB is designed to reduce the barriers to learning more about open science practices, which can be daunting for many.

\section{Make it Normative}

Making open science practices possible and easy will allow for their adoption. But we are social animals who learn and take cues from mentors and colleagues. Traditionally, that has been in striving for publications in the highest impact journals, but that does not align with any particular scientific ideal. Seeing others practice various Mertonian norms will contradict the notion that these idealistic behaviors are rarely conducted. Making them impossible to ignore only helps that along. Open Science Badges (cos.io/badges) are a means to that end (see Figure 2). Offering these visible indicators of data sharing, materials sharing, or preregistration is associated with an uptake of those behaviors over a period of time when overall rates of data sharing were not on the rise (Kidwell et al., 2016). Importantly, it is not just the offer of the badge that spurs uptake of the action, as a recent RCT showed (Rowhani-Farid et al., 2020). What is likely to be necessary is a long time period of 1 to 2 years and seeing these steps being taken by respected peers. In other words, it is not the badge that incentivizes the behavior, but it is the peer pressure of seeing these practices become normal that encourages adoption. Perusing through a current table of contents of the journal Psychological Science reinforces that these practices are becoming normal, as most articles have one or more badges associated with 
them, without any sort of mandate or penalty for inaction (e.g. 6 out of 10 articles in Volume 31, Number 12, December 2020 have one or more of open data, open materials, or preregistered badges).

[Figure 2]

But even more helpful is seeing an example of shared data, materials, or preregistrations from within your own discipline, to serve as a more direct inspiration and template for your own work. Recent issues of Journal of Educational Psychology, Exceptional Children, Gifted Child Quarterly, Language Learning, and Journal of Research on Educational Effectiveness provide such examples. Each badged article, particularly those from within one's own community, provides a template to emulate. This database contains a growing list of articles that have been awarded one or more of these badges:

https://www.zotero.org/groups/2146879/open science badges/library

Beyond searching for examples from peers, creating communities of practice is a direct way to improve norms within a field. The first annual Unconference on Open Scholarship Practices in Education Research was held in February, 2021 (https://osf.io/da3nf/), in which hundreds of educational researchers joined to develop skills in open research and to advocate for improved norms. These "unconferences" are opportunities to build such communities through their nontraditional formats, in which any attendee can define a problem or propose a solution and solicit support for addressing the topic. This gathering consisted of unconference sessions in which a group works toward a consensus solution to a defined problem and hackathons in which tasks were taken to begin to solve the problem, unconferences serve as a catalyst for culture change.

Finally, a critical component of generating norms is being asked by peers to comply with expectations. Peer review is a natural and expected opportunity for this to occur, and there is a least one initiative in which one can pledge to request open practices at this step, the Peer Reviewers' Openness (PRO) Initiative (https://www.opennessinitiative.org/). Importantly, this does not put an author in an impossible position by requiring transparency when it is not feasible. Rather, it provides template language to request that these steps be taken, or to offer an explanation of why it cannot be done. Helping to shift normal expectations toward transparency requires that we being to ask this of each other, and this is an ideal step to allow that to occur. 


\section{Make it Rewarding}

Beyond motivation through norms and community standards, there can be specific rewards given to researchers for desired behaviors. The three primary mechanisms for concrete rewards in the academic research community are: getting published, getting funded, and getting hired. Getting published should depend on conducting a study with interesting questions and rigorous methods, not on convincing editors or reviewers that the results are particularly surprising. When getting published depends on obtaining particular findings, authors are incentivized to find and report only the most unexpected results. Though at first this might seem innocuous - after all, such findings are the very definition of new discoveries that drive science forward- it actually leads to misapplication of statistical tools and irreproducible research practices. As discussed above, the motive to find or present only one type of finding, and the ability to do so given often unreported flexibility in data analysis, are sufficient for undermining the credibility of many findings.

How do we avoid self-interested outcome reporting and incentivize disinterested investigation? Registered Reports are one answer. This is a publishing format in which peer review occurs before results are known (Chambers et al., 2014; see Figure 3). See Reich (2021/this issue) for an excellent overview of this format.

\section{[Figure 3]}

A few common questions about the format include whether or not reviewers would be willing to spend time on this new format, and whether this disincentivizes the types of serendipitous discoveries that fuel scientific advancement. As for reviewer engagement, there is reason to suspect that being able to provide feedback that can actually improve a study before it is conducted is more satisfying than performing a post-mortem on a fatally flawed project, and early, anecdotal evidence suggests that reviewers do in fact respond positively to the opportunity to provide such important and timely feedback (DeHaven et al., 2019). As with the other ways that peer reviewers can encourage better practices (see the PRO Initiative mentioned above), Registered Reports offer a concrete path for journals, authors, and reviewers to address publication bias and ensure that open practices are recognized through publication. The hundreds of adopting journals and completed studies in just the past few years also suggest that reviewers are willing to engage with the format. 
In regards to the possibility that this format discourages serendipity, there is no reason to suspect this to be true, for two reasons. First, authors are specifically encouraged to report the results of unplanned analyses in their manuscripts. If an unexpected covariate leads to a difference that was not predicted, they can report those findings as exploratory conclusions that deserve to be confirmed through later, confirmatory research. Second, this process of discovery will simply always be rewarded as a basic motivator for researchers who seek to be first to discover unexpected trends in the world.

Preliminary research on the format indicates that it is working as intended. In contrast with other such efforts, Registered Reports appear to result in credible, null findings more frequently than comparable studies (Scheel et. al., 2020). Notably, there does not yet appear to be a change in citation rates for the format (Hummer et al., 2017). Though citation rates are not indicative of quality or importance (Cagan, 2013), they can be important indices - and as such are studied accordingly.

The second major reward opportunity in academia is getting funded. As with Registered Reports, funding review does typically occur before study results are known. However, the work that occurs throughout the life of a grant is subject to the same pressures and flexibility as described above. Funders can still incentivize open science practices by evaluating research proposals based on the inclusion of data management plans that include sharing, plans to preregister studies, or even partner with a journal to jointly decide to fund and publish results before result are known (https://cos.io/top-funders). These steps may be especially critical at shifting expectations, and steps by funders of education research are already underway (see below for more examples, ie SEER standards).

Finally, getting hired can and should depend on the degree to which a scholar follows practices that align with the widely endorsed Mertonian norms. There is a growing movement to request that job applicants include their adoption of practices such as sharing data or preregistering their studies. Schönbrodt (2019) has collected many of these announcements on the OSF for other hiring committees to use (https://osf.io/7jbnt/). These practices can be used or adopted by others hoping to directly mention these practices in job ads or promotion considerations.

\section{Make it Required}

When open science practices are widely possible, easy, normal, and rewarded, there will be little left to do to ensure that the research community acts in accordance with these norms. 
However, formalizing these norms through policies and requirements can help clarify expectations and address edge cases or exceptions to these norms. The Transparency and Openness Promotion (TOP) Guidelines provide a roadmap for achieving this goal. Developed in 2014 among a group of social scientists, editors, and funders, the TOP Guidelines (https://cos.io/top) cover eight specific standards where open science can be practiced in publishing and funding (Nosek et al., 2015). These include data citation; data, materials, and code transparency; design and analysis transparency; study and analysis plan preregistration; and replication research. Each of the eight standards can be applied in three levels of increasing rigor: Level 1, Disclosure of a practice; Level 2, a mandate for transparency; and Level 3, Verification or Computational Reproducibility (a fourth level, 0, represents the infective status quo and is not compliant with TOP). TOP provides journals and funders with specific recommendations for the policies that they wish to implement and a plan for increasing rigor in the future.

Education researchers are increasingly encountering these types of requirements. The Institute for Education Sciences in the U.S. Department of Education, for example, is implementing more strict guidelines for what type of open practices, such as data transparency or preregistration. These Standards for Excellence in Education Research (https://ies.ed.gov/seer/index.asp) provide recommendations or, in some cases, requirements for such practices. Other funders, including those that support education research, are taking similar steps. A curated list of funder policies regarding open science practices is available at cos.io/top-funders.

\section{Culture Change Among Policy Makers}

The above roadmap for changing culture has largely been applied to the relatively broad audience of academic researchers. However, this same strategy can be applied to a narrower community, as well: policy makers. The decision makers in academia include those who set policy at journals, funding agencies, and universities. Journals are an interesting case study, as their policy-making is widely variable. Some are fully owned by commercial publishers, who control all editorial policies. Some are published by commercial publishers on behalf of academic societies, and so editorial policies are often jointly implemented. Others are fully society owned and operated, with publications committees in charge of policies. Finally, some journals are a "one person shop," with all control in the hands of a single editor. In any of these cases, the same strategy of making change possible, easy, normative, rewarding, and then required can occur. 
Implementation of TOP Guidelines themselves are following this same pattern. Published in 2015, TOP made policy change easy by providing specific room for improvement and policy language to do so. This was followed by a signatory campaign that garnered massive support among journals, academic societies, publishers, and funders, with over 5,000 such entities becoming a signatory. This normalized the TOP Guidelines and built a community of supporters. Now the process of rewarding implementation of the TOP Guidelines with TOP Factor begins. TOP Factor is a direct alternative to journal metrics that evaluate novelty or attention around research, most notably the Journal Impact Factor. Widely acknowledged as incentivizing poor research practices (see the Declaration of Research Assessment, https://sfdora.org/), JIF is misused by applying measures of attention as an indicator of research (and by extension, researcher) quality. TOP Factor refocuses attention on journal practices by giving points to the level of adoption for each of the eight standards (plus whether or not the journal uses Registered Reports or Open Science Badges). Zero points are assigned for journals that do not use a TOP-compliant policy (for example, they do not mention data transparency, or merely encourage it), 1 point is assigned for a policy that complies with TOP Level 1 (for example, requiring a data availability statement), 2 points for a mandate for data transparency (with exceptions only permitted for legal or ethical constraints), or 3 for computational reproducibility checks. The standard for preregistration likewise awards 1 point for disclosure of whether or not work was preregistered, 2 points for verifying that any preregistered work was reported transparently (i.e. by ensuring that all test outcomes were reported and any new outcomes were disclosed as exploratory only), and 3 for requiring that empirical work be preregistered. A complete rubric for evaluating journal policies is available at https://osf.io/t2yu5/, and Figure 4 provides an overview of the database interface.

[Figure 4]

With 683 journals currently evaluated (as of January, 2021), too many publications are taking too few steps to address transparency and reproducibility (the data behind these scores are available at: https://osf.io/qatkz/). This means that educational psychology journals have a great opportunity to be among the early adopters in the field of education in adopting these practices. Given the adoptions that have already occurred in other branches of psychology, editors, 
publishers, and editorial committees at educational psychology journals have good models from which to develop their own guidelines and procedures. Editors can filter policies by the topic that is easiest to engage with (for example, materials and code sharing does not have the same complications as does data sharing) and model their policies based on those with "Level 2" (mandate transparency) approach to those topics, while improving their data transparency policy by requiring specific disclosure of how data are (or are not) accessible with a "Level 1" policy.

Use of the "TOP Statement" (a standardized disclosure of open practices questionnaire, see https://osf.io/sm78t/) can simplify enforcement of this type of policy. Such a change in policy would comply with funder expectations for open science practices, including the U.S. Department of Education's Institute for Education Science's Standards for Excellence in Education Research (SEER) https://ies.ed.gov/seer/index.asp.

With a mean TOP Factor score of 3.36 and median of 1 , most peer reviewed journals are not even taking minimal steps of disclosure to show whether or not underlying evidence is available for peers to review and verify (see Figure 5). However, for those that are taking these steps, there is ample opportunity to give recognition and credit for such steps. For education researchers who want to submit to such journals, the database of these journal policies (www.topfactor.org) provide a place to find relevant journals that are taking concrete steps in the right direction. And for decision makers at journals or publishers, they can find examples of relevant journals taking these actions and emulate their steps in their own author guidelines and procedures.

[Figure 5]

[Table 1]

\section{The Emerging Future of Open, Reproducible Research in Educational Psychology}

We are at a crossroads in scientific research, perhaps even one of Kuhn's Revolutions. None of the above practices, by themselves, are revolutionary or even particularly new. Indeed, the motto of the esteemed Royal Society, "Nullius in verba" ("On the word of no one" or "Take nobody's word for it"), suggests that verification, only possible through transparency, is an 
essential element of the process of science. However, it is only recently that we have had the means to enable the type of transparency needed to ensure that this verification is readily possible.

Table 1 provides specific recommendations for working towards improved research culture. Individual researchers have the opportunity to take important steps to become models for emulation and to help begin conversations in their departments. Policy makers at universities, journals, or academic societies can take steps that will have wide reach among their communities, such as adopting policies that specifically reward the types of behaviors the scientific community values. Most readers will be able to adopt one or more of the steps with relative ease. Taking that action for a few subsequent projects will refine that skill and prepare the researcher for some other step that piques their interest or is requested of them. Meanwhile, each step serves as a demonstration to learn from one's peers and does its part to shift norms. Other readers will be in a position to affect policy. Each step represents an opportunity to raise the issue with constituent authors or grantees. Asking one to disclose whether or not data are openly available, or whether the work was preregistered, reminds the author to consider that action for next time. It has the further benefit of helping the reader to easily determine the answer to that question themselves (for example if the article has a badge indicating that data or preregistrations are available). Shifting peer review to before the study is conducted allows for proposed designs to be improved prior to conducting them, and can reduce the bias against null findings.

The scientific community agrees that the status quo runs counter to the ideal process by which research should proceed (Anderson et al., 2007). I hope that every player in the community will showcase steps that they are taking to be more transparent or provide a platform for these practices to occur in their university or on the pages of their journals. I invite every reader to consider how their next study will include one of the open practices covered in this special issue, and every policy maker at a journal, academic society, or university to consider ways to recognize and reward these practices. 


\section{Literature Cited}

Aalbersberg, Ij. J., Appleyard, T., Brookhart, S., Carpenter, T., Clarke, M., Curry, S., Dahl, J., DeHaven, A. C., Eich, E., Franko, M., Freedman, L., Graf, C., Grant, S., Hanson, B., Joseph, H., Kiermer, V., Kramer, B., Kraut, A., Karn, R. K., .. Vazire, S. (2018). Making Science Transparent By Default; Introducing the TOP Statement [Preprint]. Open Science Framework. https://doi.org/10.31219/osf.io/sm78t

Agnoli, F., Wicherts, J. M., Veldkamp, C. L. S., Albiero, P., \& Cubelli, R. (2017). Questionable research practices among italian research psychologists. PLOS ONE, 12(3), e0172792. https://doi.org/10/f9twdr

Anderson, M. S., Martinson, B. C., \& De Vries, R. (2007). Normative Dissonance in Science: Results from a National Survey of U.S. Scientists. Journal of Empirical Research on Human Research Ethics: An International Journal, 2(4), 3-14. https://doi.org/10/cfs74f

Camerer, C. F., Dreber, A., Forsell, E., Ho, T.-H., Huber, J., Johannesson, M., Kirchler, M., Almenberg, J., Altmejd, A., Chan, T., Heikensten, E., Holzmeister, F., Imai, T., Isaksson, S., Nave, G., Pfeiffer, T., Razen, M., \& Wu, H. (2016). Evaluating replicability of laboratory experiments in economics. Science, 351(6280), 1433-1436. https://doi.org/10/bdps

Camerer, Colin F., Dreber, A., Holzmeister, F., Ho, T.-H., Huber, J., Johannesson, M., Kirchler, M., Nave, G., Nosek, B. A., Pfeiffer, T., Altmejd, A., Buttrick, N., Chan, T., Chen, Y., Forsell, E., Gampa, A., Heikensten, E., Hummer, L., Imai, T., ... Wu, H. (2018). Evaluating the replicability of social science experiments in Nature and Science between 2010 and 2015. Nature Human Behaviour, 1. https://doi.org/10/gd3v2n

Cagan, R. (2013). The San Francisco Declaration on Research Assessment. Disease Models and Mechanisms, 6(4), 869. https://doi.org/10.1242/dmm.012955

Chambers, C., Feredoes, E., D. Muthukumaraswamy, S., J. Etchells, P., \& 1 Cardiff University Brain Research Imaging Centre, School of Psychology, Cardiff University; (2014). Instead of "playing the game" it is time to change the rules: Registered Reports at AIMS Neuroscience and beyond. AIMS Neuroscience, 1(1), 4-17. https://doi.org/10/gdnbt7

Chang, A. C., \& Li, P. (2015). Is Economics Research Replicable? Sixty Published Papers from Thirteen Journals Say "Usually Not." Finance and Economics Discussion Series, 2015(83), 1-26. https://doi.org/10/gfgv79

Committee on Reproducibility and Replicability in Science. (2019). Reproducibility and Replicability in Science (p. 25303). National Academies Press. https://doi.org/10.17226/25303

Cova, F., Strickland, B., Abatista, A., Allard, A., Andow, J., Attie, M., Beebe, J., Berniūnas, R., Boudesseul, J., Colombo, M., Cushman, F., Diaz, R., N'Djaye Nikolai van Dongen, 
N., Dranseika, V., Earp, B. D., Torres, A. G., Hannikainen, I., Hernández-Conde, J. V., Hu, W., ... Zhou, X. (2018). Estimating the Reproducibility of Experimental Philosophy. Review of Philosophy and Psychology. https://doi.org/10/gf28gh

DeHaven, A. C., Graf, C., Mellor, D. T., Morris, E., Moylan, E., Pedder, S., \& Tan, S. (2019, September 17). Registered Reports: views from editors, reviewers and authors. https://doi.org/10.31222/osf.io/ndvek

Fleming, J. I., Wilson, S. E., Hart, S. A., Therrien, W. J., \& Cook, B. G. (2021/this issue). Open accessibility in education research: Enhancing the credibility, equity, impact, and efficiency of research. Educational Psychologist, 56(2), \#\#-\#\#.

Fraser, H., Parker, T., Nakagawa, S., Barnett, A., \& Fidler, F. (2018). Questionable research practices in ecology and evolution. PLOS ONE, 13(7), e0200303. https://doi.org/10/gdtmg2

Gabelica, M., Cavar, J., \& Puljak, L. (2019). Authors of trials from high-ranking anesthesiology journals were not willing to share raw data. Journal of Clinical Epidemiology, 0(0). https://doi.org/10/gfvpwc

Gehlbach, H., \& Robinson, C. D. (2021/this issue). From old school to open science: The implications of new research norms for educational psychology and beyond. Educational Psychologist, 56(2), \#\#\#-\#\#

Hagger, M. S., Chatzisarantis, N. L. D., Alberts, H., Anggono, C. O., Batailler, C., Birt, A. R., Brand, R., Brandt, M. J., Brewer, G., Bruyneel, S., Calvillo, D. P., Campbell, W. K., Cannon, P. R., Carlucci, M., Carruth, N. P., Cheung, T., Crowell, A., De Ridder, D. T. D., Dewitte, S., ... Zwienenberg, M. (2016). A Multilab Preregistered Replication of the Ego-Depletion Effect. Perspectives on Psychological Science, 11(4), 546-573. https://doi.org/10.1177/1745691616652873

Hardwicke, T. E., Mathur, M. B., MacDonald, K., Nilsonne, G., Banks, G. C., Kidwell, M. C., Hofelich Mohr, A., Clayton, E., Yoon, E. J., Henry Tessler, M., Lenne, R. L., Altman, S., Long, B., \& Frank, M. C. (2018). Data availability, reusability, and analytic reproducibility: Evaluating the impact of a mandatory open data policy at the journal Cognition. Royal Society Open Science, 5(8), 180448. https://doi.org/10.1098/rsos.180448

Helmreich, R. L., Merritt, A. C., \& Wilhelm, J. A. (1999). The Evolution of Crew Resource Management Training in Commercial Aviation. The International Journal of Aviation Psychology, 9(1), 19-32. https://doi.org/10.1207/s15327108ijap0901 2

Hummer, L. T., Singleton Thorn, F., Nosek, B. A., \& Errington, T. M. (2017, December 4). Evaluating Registered Reports: A Naturalistic Comparative Study of Article Impact. https://doi.org/10.31219/osf.io/5y8w7

loannidis, J. P. A. (2005). Why Most Published Research Findings Are False. PLoS Medicine, 2(8), e124. https://doi.org/10/chhf6b 
loannidis, J. P. A., Allison, D. B., Ball, C. A., Coulibaly, I., Cui, X., Culhane, A. C., Falchi, M., Furlanello, C., Game, L., Jurman, G., Mangion, J., Mehta, T., Nitzberg, M., Page, G. P., Petretto, E., \& van Noort, V. (2009). Repeatability of published microarray gene expression analyses. Nature Genetics, 41(2), 149-155. https://doi.org/10/bcn2tr

John, L. K., Loewenstein, G., \& Prelec, D. (2012). Measuring the Prevalence of Questionable Research Practices With Incentives for Truth Telling. Psychological Science, 23(5), 524-532. https://doi.org/10/f33h6z

Kidwell, M. C., Lazarević, L. B., Baranski, E., Hardwicke, T. E., Piechowski, S., Falkenberg, L.-S., Kennett, C., Slowik, A., Sonnleitner, C., Hess-Holden, C., Errington, T. M., Fiedler, S., \& Nosek, B. A. (2016). Badges to Acknowledge Open Practices: A Simple, Low-Cost, Effective Method for Increasing Transparency. PLOS Biol, 14(5), e1002456. https://doi.org/10/f8pkck

Lee, C. J., \& Moher, D. (2017). Promote scientific integrity via journal peer review data. Science, 357(6348), 256-257. https://doi.org/10/ggb759

Makel, M. C., Plucker, J. A., \& Hegarty, B. (2012). Replications in Psychology Research: How Often Do They Really Occur? Perspectives on Psychological Science: A Journal of the Association for Psychological Science, 7(6), 537-542. https://doi.org/10/f4fdbt

Merton, R. (1973). The Normative Structure of Science. In The Sociology of Science: Theoretical and Empirical Investigations (pp. 267-278). University of Chicago Press.

Meyer, M. N. (2018). Practical Tips for Ethical Data Sharing. Advances in Methods and Practices in Psychological Science, 1(1), 131-144. https://doi.org/10.1177/2515245917747656

Nosek, B. A., Alter, G., Banks, G. C., Borsboom, D., Bowman, S. D., Breckler, S. J., Buck, S., Chambers, C. D., Chin, G., Christensen, G., Contestabile, M., Dafoe, A., Eich, E., Freese, J., Glennerster, R., Goroff, D., Green, D. P., Hesse, B., Humphreys, M., ... Yarkoni, T. (2015). Promoting an open research culture. Science, 348(6242), 14221425. https://doi.org/10/gcpzwn

Nosek, B. A., \& Errington, T. M. (2020). What is replication? PLOS Biology, 18(3), e3000691. https://doi.org/10.1371/journal.pbio.3000691

Nosek, B. (2019). Strategy for Culture Change. Strategy for Culture Change. https://cos.io/blog/strategy-culture-change/

Nosek, B. A., Spies, J. R., \& Motyl, M. (2012). Scientific Utopia: II. Restructuring Incentives and Practices to Promote Truth Over Publishability. Perspectives on Psychological Science, 7(6), 615-631. https://doi.org/10/f4fc2k

Open Science Collaboration. (2015). Estimating the reproducibility of psychological science. Science, 349(6251), aac4716. https://doi.org/10/68c

Plucker, J. A., \& Makel, M. C. (2021/this issue). Replication is important for educational psychology: Recent developments and key issues. Educational Psychologist, 56(2), \#\#\#-\#\#. 
Rabelo, A. L. A., Farias, J. E. M., Sarmet, M. M., Joaquim, T. C. R., Hoersting, R. C., Victorino, L., Modesto, J. G. N., \& Pilati, R. (2019). Questionable research practices among Brazilian psychological researchers: Results from a replication study and an international comparison. International Journal of Psychology, n/a(n/a). https://doi.org/10.1002/ijop.12632

Reich, J. (2021/this issue). Pre-Registration and Registered Reports. Educational Psychologist, 56(2), \#\#\#-\#\#.

Rogers, E. (1963). Diffusion of Innovations. Free Press of Glencoe.

Rowhani-Farid, A., Aldcroft, A., \& Barnett, A. G. (2020). Did awarding badges increase data sharing in BMJ Open? A randomized controlled trial. Royal Society Open Science, 7(3), 191818. https://doi.org/10.1098/rsos.191818

Scheel, A. M., Schijen, M., \& Lakens, D. (2020, February 5). An excess of positive results: Comparing the standard Psychology literature with Registered Reports. https://doi.org/10.31234/osf.io/p6e9c

Schönbrodt, F. D., Mellor, D. T., Bergmann, C., Penfold, N., \& Westwood, S. J. (2020, June 15). Academic job offers that mentioned open science. Retrieved from osf.io/7jbnt

Silberzahn, R., Uhlmann, E. L., Martin, D. P., Anselmi, P., Aust, F., Awtrey, E., Bahník, Š., Bai, F., Bannard, C., Bonnier, E., Carlsson, R., Cheung, F., Christensen, G., Clay, R., Craig, M. A., Dalla Rosa, A., Dam, L., Evans, M. H., Flores Cervantes, I., ... Nosek, B. A. (2018). Many Analysts, One Data Set: Making Transparent How Variations in Analytic Choices Affect Results. Advances in Methods and Practices in Psychological Science, 1(3), 337-356. https://doi.org/10.1177/2515245917747646

Simmons, J. P., Nelson, L. D., \& Simonsohn, U. (2011). False-Positive Psychology: Undisclosed Flexibility in Data Collection and Analysis Allows Presenting Anything as Significant. Psychological Science, 22(11), 1359-1366. https://doi.org/10/bxbw3c

Stodden, V., Seiler, J., \& Ma, Z. (2018). An empirical analysis of journal policy effectiveness for computational reproducibility. Proceedings of the National Academy of Sciences, 115(11), 2584-2589. https://doi.org/10/gc8gkw 


\section{Changing a Research Culture}

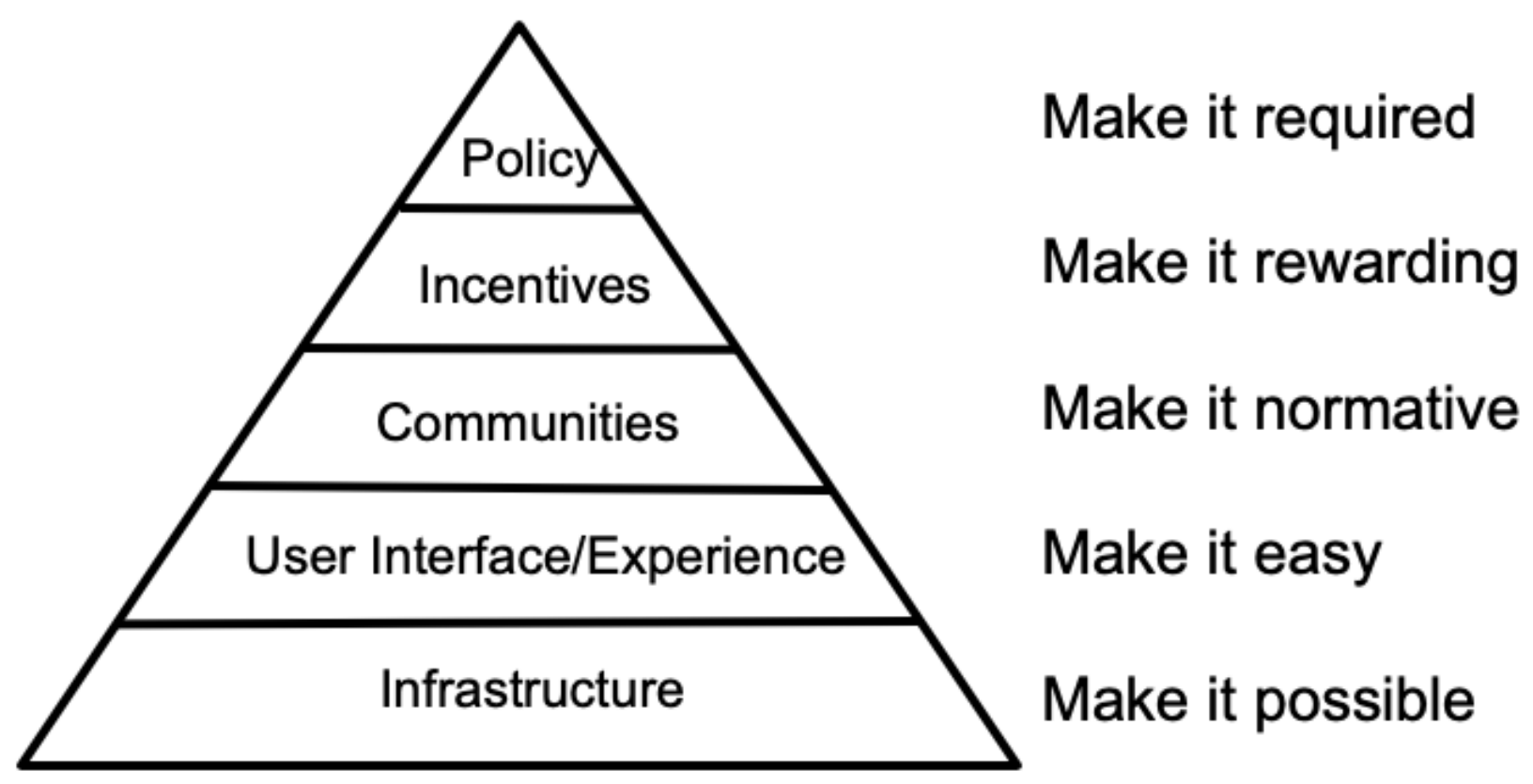

Figure 1: A strategy for culture change.

Caption: The strategy for culture change requires building tools to make change possible, makingthing them easy to use, fostering communities of practice, incentivizing ideal behaviors, and finally making them required when consensus is possible. (Nosek, 2019) 

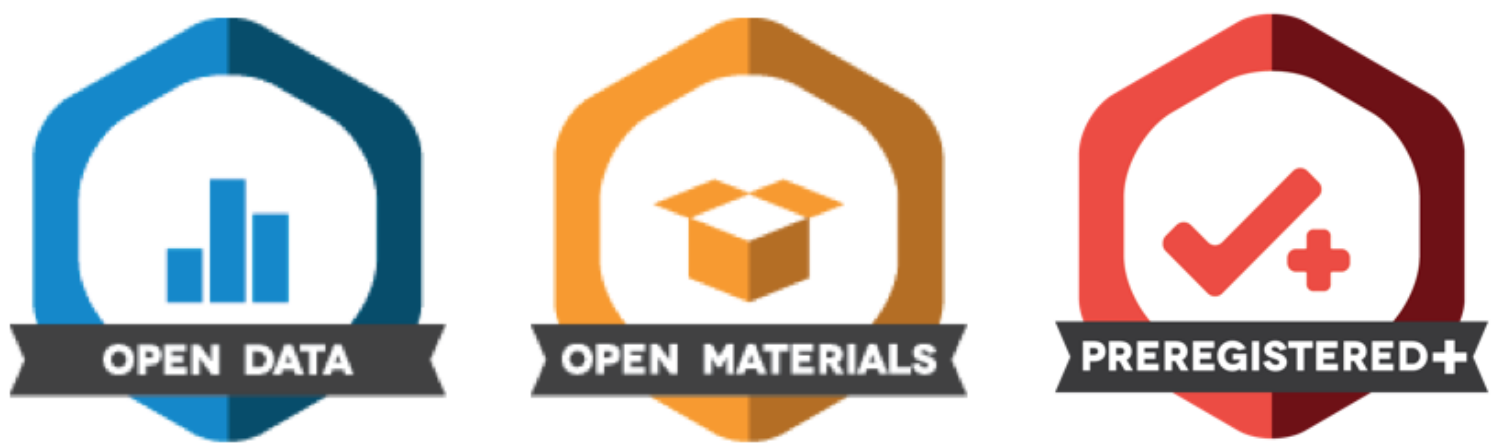

Figure 2: Open Science Badges.

Caption: These icons serve as an indicator that norms are changing within a community.

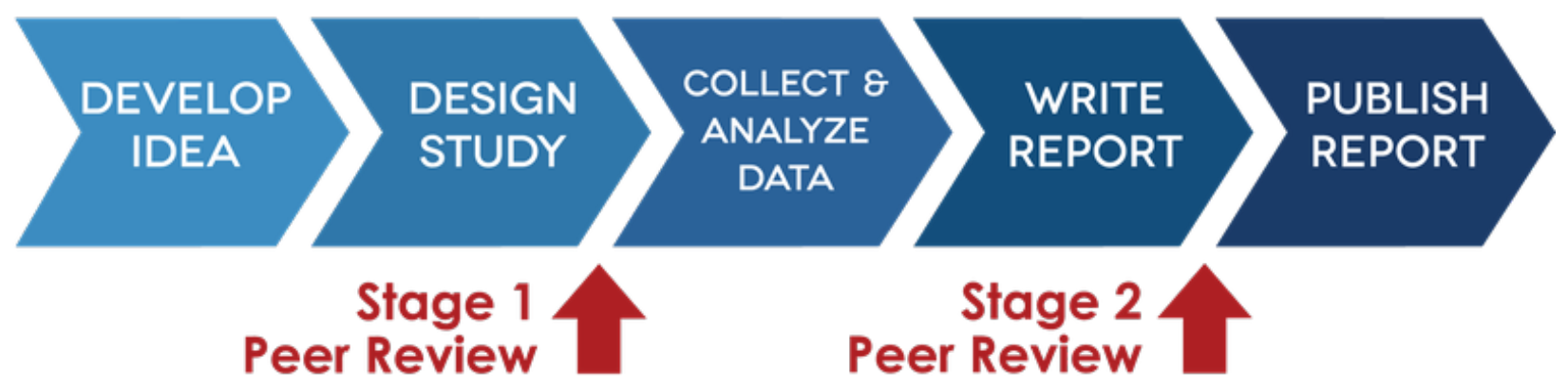

Figure 3: Registered Reports.

Caption: This format changes the process of peer review, and evaluates research questions and methods without knowing how the results will turn out (cos.io/rr). 


\begin{tabular}{|c|c|c|c|c|c|c|c|c|}
\hline \multicolumn{5}{|l|}{ A project by Center for Open Science } & & \multicolumn{3}{|c|}{$\begin{array}{l}\text { TOP GUIDELINES } \\
\text { NCY AND OPENNESS PROMOTION }\end{array}$} \\
\hline TOP Standards (3) & Search Journal Titles & & & & & & & \\
\hline $\begin{array}{l}\text { 『 Data Citation } \\
\nabla \quad \text { Data Transparency }\end{array}$ & Journal & $\downarrow$ Total & $\begin{array}{r}\text { Data } \\
\text { Citation }\end{array}$ & $\begin{aligned} \begin{array}{l}\text { Data } \\
\text { Transparency }\end{array} & \end{aligned}$ & $\begin{array}{r}\begin{array}{r}\text { Analysis } \\
\text { Code } \\
\text { Transparency }\end{array} \\
\end{array}$ & $\begin{array}{r}\text { Materials } \\
\text { Transparency }\end{array}$ & $\begin{array}{l}\text { Design \& } \\
\text { Analysis } \\
\text { Reporting } \\
\text { Guidelines }\end{array}$ & $\begin{array}{r}\text { Study } \\
\text { Preregistration }\end{array}$ \\
\hline$\checkmark \quad$ Analysis Code Transparency & $\begin{array}{l}\text { Gifted Child Quarterly } \llbracket \\
\text { SAGE }\end{array}$ & 16 & 2 & 1 & 1 & 1 & 1 & 1 \\
\hline $\begin{array}{l}\text { Design \& Analysis Reporting } \\
\text { Guidelines }\end{array}$ & $\begin{array}{l}\text { Exceptional Children } \square \\
\text { SAGE }\end{array}$ & 15 & 1 & 1 & 1 & 1 & 1 & 1 \\
\hline$\checkmark$ Study Preregistration & $\begin{array}{l}\text { Royal Society Open Sci- } \\
\text { ence } \mathbb{2} \\
\text { Royal Society Publishing }\end{array}$ & 14 & 2 & 2 & 2 & 2 & 0 & 0 \\
\hline \multirow{3}{*}{$\begin{array}{ll}\nabla & \text { Analysis Plan Preregistration } \\
\nabla & \text { Replication } \\
& \text { Registered Reports \& } \\
\text { Publication Bias }\end{array}$} & $\begin{array}{l}\text { Language Learning } \llbracket \\
\text { Wiley }\end{array}$ & 11 & 2 & $0^{\circ}$ & $0^{\circ}$ & $0^{\circ}$ & $i$ & 0 \\
\hline & $\begin{array}{l}\text { Science } t \\
\text { AAAS }\end{array}$ & 11 & 2 & 2 & 2 & $i$ & 1 & 1 \\
\hline & $\begin{array}{l}\text { PLOS ONE [ } \\
\text { PLOS }\end{array}$ & 11 & 1 & 2 & $0^{\circ}$ & $0^{\circ}$ & 2 & 0 \\
\hline$\checkmark \quad$ Open Science Badges & $\begin{array}{l}\text { Child Development } \llbracket \\
\text { Wiley }\end{array}$ & 9 & $i$ & i & $i$ & i & $i$ & 1 \\
\hline \multirow[t]{6}{*}{ Publishers } & $\begin{array}{l}\text { Journal of Educational } \\
\text { Psychology } \square \\
\text { American Psychological } \\
\text { Associatition }\end{array}$ & 9 & 1 & $0^{\circ}$ & 0 & 0 & 1 & 1 \\
\hline & $\begin{array}{l}\text { Proceedings of the Nation- } \\
\text { al Academy of Sciences } \\
\text { U.S National Academy of } \\
\text { Sciences }\end{array}$ & 8 & 1 & $i$ & 1 & 1 & 1 & $i$ \\
\hline & $\begin{array}{l}\text { Learning and Instruc- } \\
\text { tion } \underbrace{-} \\
\text { Elsevier }\end{array}$ & 7 & 1 & $0^{\circ}$ & 0 & 0 & 0 & 0 \\
\hline & $\begin{array}{l}\text { Research Synthesis Meth- } \\
\text { ods 『 } \\
\text { Wiley }\end{array}$ & 5 & 2 & $i$ & $i^{\circ}$ & 0 & 0 & 0 \\
\hline & $\begin{array}{l}\text { Scientific Studies of Read- } \\
\text { ing } 4 \text { tand francis } \\
\text { Taylor and }\end{array}$ & 4 & 1 & $0^{\circ}$ & 0 & 0 & 0 & 0 \\
\hline & $\begin{array}{l}\text { Journal of Research on Ed- } \\
\text { ucational Effectiveness } \square\end{array}$ & 3 & 1 & $0^{\circ}$ & 0 & 0 & 0 & 0 \\
\hline
\end{tabular}

Figure 4: TOP Factor Rations for Education Journals

Caption: TOP Factor rates journal policies as they relate to open and reproducible practices: https://www.topfactor.org. The included ratings are for some highly rated education journals. 


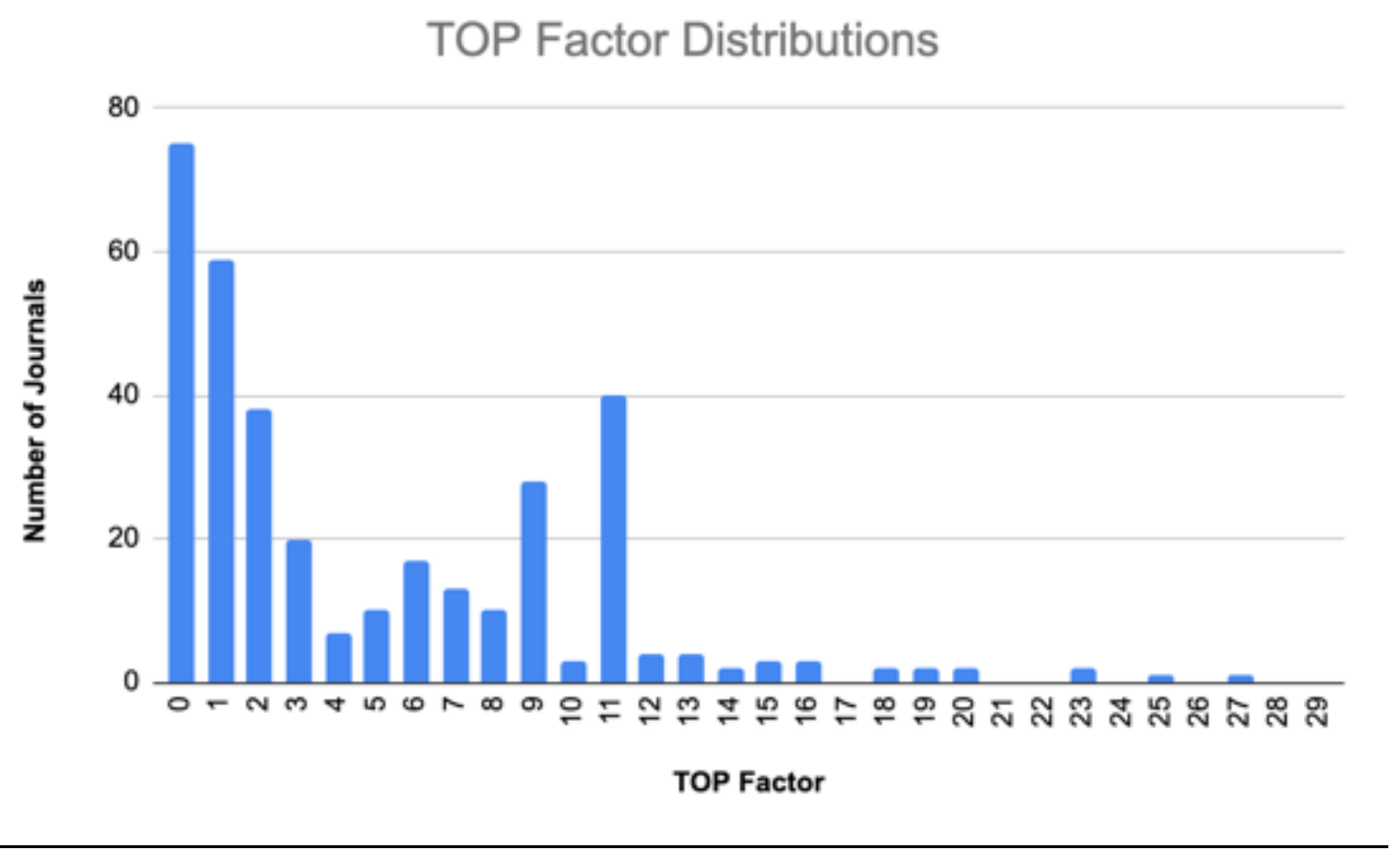

Figure 5: TOP Factor Distribution.

Caption: The ratings show that there is ample room for improvement. Our of a possible score of 29 points, most journals rate at 0 or 1 . Typically, this indicates that they may encourage data sharing, if they make any statement, but that they do not require disclosure of data availability or other open science practices. For comparison, a journal that required disclosure of data, materials, and code availability, that pointed authors to reporting and data citation guidelines, that encouraged replication studies, and that asked authors to indicate if they work was preregistered with an analysis plan would receive a rating of 8 . 